\title{
Scanning Electron Microscopy Study of the Activation of Porous Stainless Steel for Pd Electroless Plating
}

\author{
K. Munjeri, M. J. Myers, and T. Gutu \\ University of Zimbabwe, Department of Physics, Mount Pleasant, Harare, Zimbabwe
}

Activation is a fundamentally key step that produces catalytic active sites onto the surface of substrates to initiate and enhance electroless plating of metals such as platinum group metals (e.g. Pd) [1,2]. For good adhesion between the deposited metal and the substrate, the thermal expansion coefficient of the substrate should be similar to that of the metal. For example, in $\mathrm{H}_{2}$ permselective Pd-based membranes, porous stainless steel (PSS) is widely utilized as the substrate or support. In order to reduce membrane cost while still retaining high $\mathrm{H}_{2}$ selectivity and high permeation rate, thin defect-free Pd films are required [3]. However, the density of nucleation sites (seeds) on activated surface determines the minimum thickness, surface coverage, and adhesion properties of the electroless deposited Pd film [4]. Thus, the objective of this research is to study the activation of PSS by introducing Pd nuclei onto the PSS surface through a simple, inexpensive dip coating technique.

The PSS substrates were ultrasonic cleaned in an alkaline bath at $60{ }^{\circ} \mathrm{C}$, then rinsed with tape water followed by rinsing with deionized water and finally ultrasonic cleaned in isopropanol. The cleaned PSS substrates were dip coated in a seed solution of Pd Acetate in chloroform before drying at $55{ }^{\circ} \mathrm{C}$. Reduction was carried out by submersion in ultra-pure water and then adding a suitable reducing agent. Hydrogen gas and sodium hypophosphite reducing agents were used to study their effect on the activation (i.e. seeding). A JEOL JSM 7001F FESEM equipped with an Oxford EDS spectrometer was utilized to study the morphology and elemental composition of the activated PSS substrates at a working distance of about $5 \mathrm{~mm}$.

Fig. 1 shows FESEM results on the activation of PSS supports using different reducing agents and different number of seeding i.e. activation step carried out more than once. When $\mathrm{H}_{2}$ was utilized as the reducing agent, $\mathrm{Pd}$ seeds (i.e. Pd nuclei or catalytic sites) were observed as discrete white dots on SEM images even for PSS supports seeded only once as shown in Fig. 1a. The presence of Pd was revealed by Energy Dispersive X-ray Spectroscopy (EDS) analysis (results not shown) or by utilizing SEM BackScattered Electron (BSE) imaging. In Fig. 1b, the BSE image indicates the high Z Pd seeds as a white surface against the dark background of low Z Fe support. Fig. 1c-d show the SEM micrographs when sodium hypophosphite was used as the reducing agent. The Pd seeds were not observed on SEM micrographs when the PSS supports were seeded once (Fig. 1c). EDS analysis did not show the presence of Pd on the supports seeded only once. However, when the supports were seeded several times Pd seeds were easily seen on the SEM micrographs and EDS analysis revealed the presence of Pd. In Fig. 1d, Pd seeds appear as a uniform layer on the surface of the PSS support. The surface density of Pd seeds was observed to increase with the increase in the number of seeding. The morphology of the Pd seeds strongly depended on the type of the reducing agent used. A more granular surface morphology was obtained when $\mathrm{H}_{2}$ was used as the reducing agent. To conclude, this research study showed that the type of reducing agent used during the activation step strongly affected the morphology and surface density of the Pd seeds on the PSS supports. $\mathrm{H}_{2}$ resulted in immediate nucleation of discrete Pd seeds while sodium hypophosphite required several seeding steps for $\mathrm{Pd}$ seeds to nucleate. Irrespective of the reducing agent used, the Pd seeds surface density increased with the number of seeding. 
References:

[1] E Matijevic, et al., Plating and Surface Finishing 62, (1975), 958.

[2] S N Paglieri, et al, Industrial \& Engineering Chemical Research 38, (1999), 1925.

[3] S Liguori, et al, Membranes 4, (2014), 143.

[4] M Schlesinger and J. Kisel, Journal of The Electrochemical Society 136, (1989) 1658.

[5] Authors acknowledge funding from the University of Zimbabwe Research Board grant No: RB36 and The World Academy of Sciences research grant agreement No: 14-108 RG/PHYS/AF/AC_I-

UNESCO FR: 324028571. The electron microscopy analysis was done at Nelson Mandela Metropolitan University's Centre for High Resolution Transmission Electron Microscope.
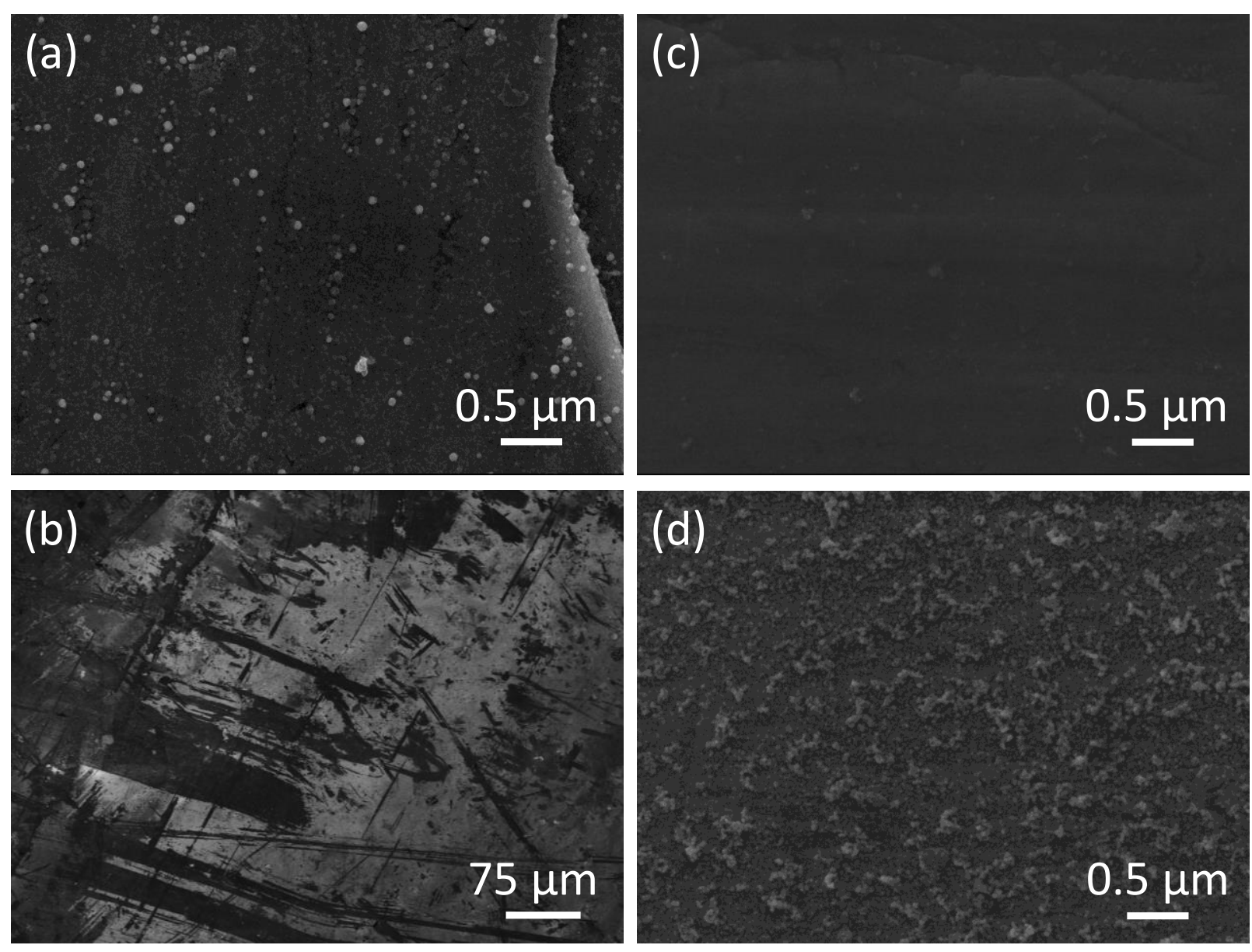

Figure 1. SEM micrographs of activated PSS supports: (a) - (b) $\mathrm{H}_{2}$ used as reducing agent, (a) seeded once, (b) BSE image, seeded five times, (c) - (d) Sodium hypophosphite used as reducing agent, (c) seeded once, and (d) seeded seven times. 